Martina Grempler (Wien/Bonn)

\title{
Ensemblebearbeitungen in der Opera buffa an den Wiener Theatern der 1760er Jahre*
}

Die Diskussion um die Ensembles nimmt in der Sekundärliteratur zum Thema Opera buffa traditionell breiten Raum ein. ${ }^{1}$ Dieser intensive Blick der Musikforschung erscheint nur allzu gerechtfertigt, denn nicht zuletzt sind es die Ensembles, die die Opera buffa des 18. Jahrhunderts von der Opera seria absetzen. Sie sind in den Worten Wolfgang Osthoffs, der die Besprechung der Ensembles mit Bedacht an das Ende seines 1973 erschienenen grundsätzlichen Beitrags zur Opera buffa stellte, für die Gattung von entscheidender Bedeutung: „Wenn die Opera buffa als musikalische Komödie ein Theater ist, das der Struktur der Gemeinschaft unterliegt, so ist es folgerichtig, daß ihre eigenste Realisation nicht in den Arien, sondern in den großen Ensembles, besonders im Finale zu suchen ist." 2

Ein großer Teil der Literatur beschäftigt sich vorwiegend mit den Ensembles der Mozart-Zeit, wobei das Finale des zweiten Aktes von Wolfgang Amadeus Mozarts Le nozze di Figaro als Musterbeispiel gilt. Besonderes Interesse erweckte außerdem das frühe 19. Jahrhundert, also die Epoche des voll ausgeprägten „Großen Finales“, 3 wofür das absurde Ende des ersten Akts von Gioachino Rossinis L'italiana in Algeri als Prototyp gelten kann. Die Literatur zur früheren Opera buffa stellte vor allem die Frage, welche Autoren als Verantwortliche für die Entwicklung komplexer Ensemblestrukturen ausgemacht werden können,

* Es handelt sich um die erweiterte Fassung eines bei der Jahrestagung der Gesellschaft für Musikforschung 2010 am Deutschen Historischen Institut in Rom gehaltenen Vortrages, der aus der Arbeit an dem an der Universität Wien angesiedelten Forschungsprojekt Die italienische Opera buffa auf der Wiener Bühne (1763-1773) erwuchs, gefördert vom Austrian Science Fund (FWF): P 21323 (Projektleiter: Michele Calella).

1 Bereits Hermann Abert, W. A. Mozart, Leipzig 1919-1921 hat sich in dem Kapitel zur Opera buffa ausführlich über die Ensembles Gedanken gemacht. Ihm folgten mit wichtigen Einzelbeiträgen etwa Herbert Schneider, „Vaudeville-Finali in Haydns Opern und ihre Vorgeschichte“, in: Bericht über den Internationalen Haydn Kongress Wien 1982, hrsg. von Eva Badura-Skoda, München 1986, S. 302309; Sabine Henze-Döhring, „La tecnica del concertato in Paisiello e Rossini“, in: NRMI 22 (1988), S. 1-23; Gerd Rienäcker, Art. „Finale“, in: MGG2, Sachteil 3, Kassel u. a. 1995, Sp. 474-488. Im angelsächsischen Raum befasste sich beginnend mit Edward J. Dent, „Ensemble and Finales in 18th Century Italian Opera“, in: SIMG 11 (1909-10), S. 543-569 sowie 12 (1910-11), S. 112-138, eine ganze Reihe von Autoren ausführlich mit den Finali, insbesondere Daniel Heartz, „The Creation of the Buffo Finale in Italian Opera", in: PRMA 104 (1977-78), S. 67-78; John Platoff, Music and Drama in the ,Opera Buffa' Finale: Mozart and His Contemporaries in Vienna, 1781-1790, Diss. Ann Arbor 1984; ders., „Musical and Dramatic Structure in the Opera Buffa Finale“, in: The Journal of Musicology 7 (1989), S. 191-230; Caryl Clark, „Ensembles and Finales“, in: The Cambridge Companion to Eighteenth-Century Opera, hrsg. von Anthony R. DelDonna und Pierpaolo Polzonetti, Cambridge 2009, S. 50-65.

2 Wolfgang Osthoff, „Die Opera buffa“, in: Gattungen der Musik in Einzeldarstellungen: Gedenkschrift Leo Schrade, hrsg. von Wulf Arlt u. a., Bern 1973, S. 678-743, hier: S. 722.

3 Vgl. zu diesem Begriff v. a. Friedrich Lippmann, „Das, Große Finale in Opera buffa und Opera seria. Paisiello und Rossini“, in: Traditionen - Neuansätze. Für Anna Amalie Abert (1906-1996), hrsg. von Klaus Hortschansky, Tutzing 1997, S. 377-398. 
wobei zwangsläufig der Name Carlo Goldoni fällt. Trotz der bereits existierenden Untersuchungen kann jedoch gerade für den Zeitraum zwischen etwa 1750 und 1780 weiterhin einiger Forschungsbedarf konstatiert werden.

Dieser Beitrag soll den Blick auf die Wiener Theater in den 1760er Jahren lenken und damit auf eine im Vergleich zur Mozart- oder Rossini-Zeit bislang weniger beachtete Epoche, die jedoch von einigem Interesse ist, da gerade zu dieser Zeit die künstlerischen Auseinandersetzungen mit dem durch Goldoni erst kurz zuvor konstituierten Modell des Dramma giocoso stattfanden, das von Italien aus nach ganz Europa exportiert wurde.

Der hier verwendete methodische Ansatz, die Ensembles unter dem Gesichtspunkt der Bearbeitungspraxis zu betrachten, bietet sich daher geradezu an und stellt ein bislang hinsichtlich der Ensembles nur selten diskutiertes Thema dar, abgesehen von Einzelfallbesprechungen, die etwa für die Prager und Wiener Version von Mozarts Don Giovanni vorliegen. ${ }^{4}$

Es kann als gewisser Common Sense gelten, dass Bearbeitungsvorgänge in der Oper sich überwiegend auf die Arien richteten, die als Solonummern für bestimmte Sänger stärker an die individuelle Besetzung einer Rolle gebunden scheinen als die Ensembles. Die Praxis, Arien auszutauschen oder neu zu komponieren und Rezitative zu verändern oder zu kürzen, jedoch das vorgegebene Gerüst aus Introduktion und Finali sowie eventuell weiteren Ensembles inmitten der Akte zu erhalten, kann als typisch für den Bereich Opera buffa gelten.

Gerade der Rahmen aus Ensemblenummern ist es, der einer Buffa neben der spezifischen Handlung überhaupt ihre Identität verleiht. Die Ensembles ermöglichten die Wiedererkennung einer Oper, wenn für einzelne Aufführungen bis zu 80 Prozent ihrer Musik verändert wurde, wie es in der damaligen Theaterpraxis durchaus Usus war.

Das ,Ensemblegerüst' bleibt erhalten, bei den Arien wird kräftig ausgetauscht - dieses Grundprinzip lässt sich insgesamt auch für die Wiener Produktionen von aus Italien importierten Opere buffe in den 1760er Jahren feststellen, selbst wenn die folgenden Ausführungen diese Erkenntnis relativieren werden.

Der vorliegende Beitrag befasst sich mit den Ensembles im Allgemeinen, sein Schwerpunkt liegt jedoch auf den Finali und vor allem den Schlussfinali, die in besonderem Maße zum Objekt von Bearbeitungen wurden. Ensembles innerhalb eines Aktes sind bei den hier untersuchten Opern noch selten anzutreffen und die Introduktionen scheinen weniger häufig von Änderungen betroffen als die Finali.

Von den 37 in Italien uraufgeführten Opern, die zwischen 1763 und 1770 auf den Wiener Spielplan gelangten, weisen fast zwei Drittel Änderungen bei den Ensembles auf. Wie bei Arien und Rezitativen findet sich dabei ein ganzes Spektrum an Bearbeitungsmöglichkeiten von kleineren Modifikationen (Abänderung der Singstimme, Instrumentation, Textänderungen, Kürzungen) über die Hinzufügung neuer Teile bis hin zur vollständigen Ersetzung eines Finales oder zur Neukomposition des ursprünglichen Librettotextes.

4 Vgl. u. a. Ian Woodfield, The Vienna Don Giovanni, Woodbridge 2010; Wolfgang Rehm, „Zur Dramaturgie von Mozarts Don Giovanni. Die beiden Fassungen Prag 1787 und Wien 1788 - ein philologisches Problem?", in: Die frühdeutsche Oper und ihre Beziehungen zu Italien, England und Frankreich. Mozart und die Oper seiner Zeit, hrsg. von Martin Ruhnke (= Hamburger Jahrbuch für Musikwissenschaft 5), Hamburg 1981, S. 247-249. Zum Thema Bearbeitungspraxis siehe insbesondere: Bearbeitungspraxis in der Oper des späten 18. Jahrhunderts, hrsg. von Ulrich Konrad, Tutzing 2007 (= Würzburger musikhistorische Beiträge 27). 


\begin{tabular}{|c|c|c|c|c|}
\hline $\begin{array}{l}\text { Wiener } \\
\text { Aufführung }\end{array}$ & Titel & Komponist & Uraufführung & $\begin{array}{l}\text { Bearbeitungen } \\
\text { bei den Ensembles }\end{array}$ \\
\hline 1763 & La conversazione & Giuseppe Scolari & Venedig 1758 & $\begin{array}{l}\text { Wegen gestrichener } \\
\text { Figuren Änderungen } \\
\text { in allen } 3 \text { Finali }\end{array}$ \\
\hline $\begin{array}{l}1763 \\
1768\end{array}$ & Il filosofo di campagna & Baldassare Galuppi & Venedig 1754 & $\begin{array}{l}\text { Kleine Korrekturen/ } \\
\text { Kürzungen }\end{array}$ \\
\hline 1764 & L'amore in musica & Antonio Boroni & Venedig 1763 & $\begin{array}{l}\text { Coro finale des } \\
\text { III. Aktes fehlt }\end{array}$ \\
\hline $\begin{array}{r}1764 \\
1768\end{array}$ & La buona figliuola & Niccolò Piccinni & Rom 1760 & $\begin{array}{l}1764 \text { wegen ge- } \\
\text { strichener Figuren } \\
\text { Änderungen in } \\
\text { allen Finali }\end{array}$ \\
\hline 1764 & $\begin{array}{l}\text { La buona figliuola } \\
\text { maritata }\end{array}$ & Niccolò Piccinni & Bologna 1761 & $\begin{array}{l}\text { Änderungen wegen } \\
\text { gestrichener Figuren }\end{array}$ \\
\hline 1765 & Li tre amanti ridicoli & Baldassare Galuppi & Venedig 1761 & $\begin{array}{l}\text { Kleinere Modifi- } \\
\text { kationen (Instrumen- } \\
\text { tation) }\end{array}$ \\
\hline 1766 & $\begin{array}{l}\text { La vedova ingegnosa o sia } \\
\text { Il medico Strambone }\end{array}$ & Giuseppe Sellitti? & Neapel 1735 & $\begin{array}{l}\text { Ersetzung des finalen } \\
\text { Duetts }\end{array}$ \\
\hline $\begin{array}{l}1767, \\
1770\end{array}$ & La contadina in corte & Antonio Sacchini & Rom 1766 ? & $\begin{array}{l}\text { Ersetzung des Duetts } \\
\text { vor dem Schlussfinale; } \\
\text { dort Einfügung eines } \\
\text { neuen Finalteils }\end{array}$ \\
\hline 1767 & Le contadine bizzarre & Niccolò Piccinni & Venedig 1763 & $\begin{array}{c}\text { Statt Duett und Coro } \\
\text { finale des III. Aktes } \\
\text { neues Finalensemble }\end{array}$ \\
\hline 1767 & Il marchese villano & Baldassare Galuppi & Venedig 1762 & $\begin{array}{l}\text { Introduzione, Finali I } \\
\text { und II ersetzt }\end{array}$ \\
\hline 1768 & La cascina & Giuseppe Scolari & Venedig 1756 & $\begin{array}{l}\text { Finale II: Akt neukom- } \\
\text { poniert } \\
\text { Finale III: Akt erwei- } \\
\text { tert }\end{array}$ \\
\hline 1768 & $\begin{array}{l}\text { Lo sposo di tre e } \\
\text { il marito di nessuna }\end{array}$ & Pasquale Anfossi & Neapel 1767 & $\begin{array}{l}\text { Finale I leicht ver- } \\
\text { ändert; Finale III: } \\
\text { Akt ersetzt; zusätzliche } \\
\text { Introduzione zum } \\
\text { III. Akt eingefügt }\end{array}$ \\
\hline 1768 & Gli uccellatori & Florian Gassmann & Venedig 1759 & $\begin{array}{l}\text { Veränderung der Intro- } \\
\text { duzione; im III. Akt } \\
\text { neues Finalensemble }\end{array}$ \\
\hline 1769 & Il barone di Torre Forte & Niccolò Piccinni & Rom 1765 & $\begin{array}{l}\text { Introduzione des } \\
\text { II. Aktes verändert }\end{array}$ \\
\hline 1769 & La pescatrice & Niccolò Piccinni & Rom 1766 & Kürzung Schlussfinale \\
\hline
\end{tabular}




\begin{tabular}{|c|c|c|c|c|}
\hline $\begin{array}{l}\text { Wiener } \\
\text { Aufführung }\end{array}$ & Titel & Komponist & Uraufführung & $\begin{array}{l}\text { Bearbeitungen } \\
\text { bei den Ensembles }\end{array}$ \\
\hline 1769 & L'isola d'amore & Antonio Sacchini & Rom 1766 & $\begin{array}{l}\text { Introduzione, Finali I } \\
\text { und II um Chöre } \\
\text { erweitert }^{6}\end{array}$ \\
\hline 1769 & La sposa fedele & $\begin{array}{l}\text { Pietro Alessandro } \\
\text { Guglielmi }\end{array}$ & Venedig 1767 & $\begin{array}{l}\text { Coro finale III. Akt } \\
\text { fehlt }^{7}\end{array}$ \\
\hline 1770 & Il ciarlone & Giuseppe Avossa & Neapel 1763 & $\begin{array}{l}\text { Änderungen / Hinzu- } \\
\text { fügungen in Finali I. } \\
\text { und II. Akt }\end{array}$ \\
\hline 1770 & Il paese della cuccagna & Baldassare Galuppi & Venedig 1750 & $\begin{array}{l}\text { Änderungen / Hinzu- } \\
\text { fügungen in allen } \\
3 \text { Finali }\end{array}$ \\
\hline 1770 & Il villano geloso & Baldassare Galuppi & Venedig 1769 & $\begin{array}{l}\text { Neues Finalensemble } \\
\text { III. Akt }\end{array}$ \\
\hline 1770 & La serva astuta & Alessandro Felici & Florenz 1768 & $\begin{array}{l}\text { Neues Finalensemble } \\
\text { III. Akt }\end{array}$ \\
\hline 1770 & Lo sposo burlato & Niccolò Piccinni & Rom 1769 & $\begin{array}{l}\text { Erweiterung Finale } \\
\text { III. Akt }\end{array}$ \\
\hline
\end{tabular}

\section{Rollenstreichungen}

Kleinere Veränderungen innerhalb eines Ensembles ergaben sich etwa, wenn für die Wiener Aufführungen die Anzahl der handelnden Personen reduziert wurde. So entfiel bei der ersten Wiener Aufführungsserie von Niccolò Piccinnis La buona figliuola 1764 sowie im selben Jahr bei La buona figliuola maritata jeweils die Figur der Paoluccia. Deren Arien konnten einfach gestrichen werden, für die Ensembles galt es, eine andere Lösung zu finden. Man entschied sich für die denkbar einfachste Variante, nämlich ihren Part einfach bestehen und von einer anderen Figur singen zu lassen.

Bei La buona figliuola übernahm im Finale des ersten Aktes die Marchesa die Zeilen von Paoluccia, im Finale des zweiten Aktes Mengotto, dessen Partizipation dort ursprünglich nicht vorgesehen war. Piccinnis Idee, Sandrina und Paoluccia in den Finali musikalisch wie dramaturgisch stets zusammenzuspannen, fiel weg und damit ein origineller Gedanke der Theaterpraxis zum Opfer. Der Einfall verliert an Wirkung, wenn es in beiden Finali unterschiedliche Personen sind, mit denen Sandrina zusammen singt, da die Paarbildung von Sandrina und Paoluccia auf der Hand lag: beide sind Dienerfiguren und agieren über

6 Die Erweiterung durch Chöre bildet einen Sonderfall in der Bearbeitungspraxis der damals in Wien gespielten Opere buffe. Eine ausführlichere Besprechung von L'isola d'amore soll deshalb nicht an dieser Stelle stattfinden.

7 In den Partituren A-Wn, Mus. Hs. 17789 sowie P-La, 44-IX-11 endet die Oper mit einem Rezitativ, an dessen Ende ausdrücklich „Fine dell'opera“ steht. Gleiches gilt für L'amore in musica (Partituren A-Wn, Mus. Hs. 18261 sowie D-Wa, 46 Alt 116, letzteres eine venezianische Kopie). Die Partitur I-Nc, 25.6.8-10, ansonsten der Wiener Fassung entsprechend (sie entstammt der Sammlung der nach Neapel verheirateten Habsburgerin Maria Carolina), enthält hingegen den Coro finale, vgl. <http://www.internetculturale.it/opencms/opencms/it/viewItemMag.jsp?case=\&id=oai\%3Awww.internetculturale.sbn.it\%2FTeca $\% 3 \mathrm{~A} 20 \% 3 \mathrm{ANT} 0000 \% 3 \mathrm{AIT} \% 5 \mathrm{C} \% 5 \mathrm{CICCU} \% 5 \mathrm{C} \% 5 \mathrm{CMSM} \% 5 \mathrm{C} \% 5$ C0159593>, 9.1.2012. Dass diese Opern bei der Aufführung wirklich mit einem Rezitativ schlossen, ist unwahrscheinlich, zumal die Libretti die Schlussnummer jeweils enthalten. 
die gesamte Oper hinweg gemeinsam als Intrigantinnen gegen die Titelfigur Cecchina. Die Lösung mit Mengotto, der eigentlich Cecchina liebt, wirkt hingegen forciert.

Die Übernahme von Paoluccias Part durch die Marchesa im ersten Akt von La buona figliuola sowie in den Aktfinali von La buona figliuola maritata bewirkte die Beteiligung einer Parte seria an den Finali, denn im Gegensatz zu Sandrina und Paoluccia gehört die Rolle der Marchesa eigentlich zu diesem Typus. In den Uraufführungsversionen beider Opern blieb sie dementsprechend von den Finalensembles ausgeschlossen, ebenso wie ihr männliches Pendant, Cavaliere Armidoro. ${ }^{8}$

Bereits Michele Calella hat darauf hingewiesen, dass in Wien die Rolle der Marchesa gegenüber der Uraufführungsversion abgewertet wurde und kaum noch als klassische Parte seria gelten konnte. ${ }^{9}$ Das im Vergleich zur Uraufführung kleinere Wiener Sängerensemble von 1764 bewirkte also einen Bruch mit den Konventionen bezüglich der Parti serie. Die Veränderung in den Finali mit der Übernahme des Parts einer Dienerfigur durch die Marchesa erscheint wie eine logische Konsequenz aus der ohnehin veränderten Rollenkonzeption.

$\mathrm{Zu}$ analogen Veränderungen kam es in Giuseppe Scolaris La conversazione, wo Marianna, ebenfalls eine Dienerfigur, gestrichen war. Im Finale des ersten Aktes hätte sie lediglich drei Zeilen zu singen gehabt, nämlich die Aufforderung an die anderen Figuren, sich zu Tisch zu begeben. In Wien wurde diese Aufgabe der „giovane spiritosa“ Lucrezia übertragen, wofür der Text verändert werden musste, da es sich bei Marianna ausdrücklich um eine „Tedesca serva“ handelte, was Gelegenheit bot, den für die Opera buffa so typischen Wortwitz in Form von verballhorntem, in diesem Fall, verdeutschtem' Italienisch zu bringen. Zu dieser Art von Komik gehörte das Spiel mit nationalen Stereotypen und so fordert Marianna die Gäste auf: 10

Star in tafola, sì signori,

no star tempo de far gritori

trinke Vain tempo star.

Im Wiener Libretto wurde der nun von Lucrezia gesungene Text in korrektes Italienisch verwandelt und es entfiel die Aufforderung zum Alkoholgenuss, sicher nicht nur aus metrischen Gründen, sondern weil es sich um ein den Deutschen zugeschriebenes Charakteristikum handelte: ${ }^{11}$

E' già in tavola signori;

Non è tempo di rumori

A mangiare e allegri star.

In der Wiener Partitur ${ }^{12}$ allerdings blieb das falsche Italienisch erhalten und die Zeilen fielen dort sinnvollerweise der als Gastgeberin fungierenden Madama Lindora zu. Was letzt-

8 Dessen Partie 1764 war bei der Wiener Aufführung von La buona figliuola gestrichen. Lediglich am Coro finale des dritten Aktes waren beide Parti serie beteiligt, weswegen die Anzahl der Singstimmen in Wien entsprechend reduziert werden musste.

9 Michele Calella, „La buona figliuola für die ,Teatri Privilegiati': Anmerkungen zur frühen Rezeption der Opera buffa in Wien“, in: Wiener Musikgeschichte. Annäherungen - Analysen - Ausblicke. Festschrift Hartmut Krones, hrsg. von Julia Bungardt u. a., Wien u. a. 2009, S. 149-170.

10 Libretto zur Uraufführung von La conversazione, S. 23. Konsultiertes Exemplar: <http://www.braidense. it/rd/00414.pdf>, 9.1.2012.

11 Libretto zur Wiener Aufführung, S. 21. Konsultiertes Exemplar: A-Wn, 641432-A.11,1 Mus.

12 A-Wn, Mus. Hs. 18042 (das erste Finale in Bd. 1, fol. 118v-154r). 
lich bei der Aufführung gesungen wurde und von wem, ist also nicht sicher, wahrscheinlich der hochitalienische Text, da weder Lucrezia noch Madama Lindora als deutschsprachige Figuren gezeichnet sind, vielleicht aber dennoch die ursprünglichen Zeilen einfach um des komischen Effekts willen, obwohl es in diesem Zusammenhang dramaturgisch unsinnig erscheint.

\section{Das Problem des III. Aktes}

La buona figliuola, La buona figliuola maritata und das Finale des ersten Aktes von La conversazione sind Beispiele für interne Veränderungen eines Ensembles, die sich aus pragmatischen Gründen ergeben. Bei einer Erweiterung oder vollständigen Ersetzung scheinen jedoch häufig ästhetische Gründe eine maßgebliche Rolle gespielt zu haben. Überdurchschnittlich betroffen waren bei den Wiener Bearbeitungen die Schlussfinali der dreiaktigen, in der Regel aus Venedig importierten Drammi giocosi. Die traditionelle Nummernkonstellation mit Duett des Hauptliebespaares, einem darauf folgenden Rezitativ sowie kurzem Coro finale forderte in einigen Fällen zur Veränderung heraus. Für Rom entstandene Werke, abgesehen von La buona figliuola grundsätzlich zweiaktige Intermezzi, die ohnehin bereits ein umfangreicheres Ensemble am Schluss aufwiesen, tangierte es konsequenterweise weniger. $^{13}$

Mehrfach wurde aus einem einfachen Coro finale ein wirkliches Ensemble, entweder durch Erweiterung wie zum Beispiel in La serva astuta, Lo sposo burlato und Il villano geloso oder durch eine vollständige Ersetzung wie in Le contadine bizzarre und Gli uccellatori. An diesen Beispielen zeigt sich ein Bestreben zur Musikalisierung der Finalhandlung: Spielte sich bei der Uraufführung die Handlungsaufösung mit dem Zusammenfinden der Liebespaare im Rezitativ ab, so geschah dies in Wien innerhalb eines Finalensembles.

Die Abänderung des Coro finale ging häufig einher mit einer Ersetzung oder auch Streichung des obligatorischen Liebesduetts. In Le contadine bizzarre stand bei der Uraufführung in Venedig zunächst ein Duett für Fiorina und Masino („Queste mane me l'ha fatte"), jeweils nach Rezitativen folgten eine Cavatina Aurettas sowie ein Duett Auretta/ Nardone und anschließend nochmals zwei Rezitative (III. Akt, Szene 13 und Beginn Szene 14), an die der vierzeilige Coro „Viva, viva d'ogni affanno“ anschloss. In Wien entfiel nahezu dieser gesamte Finalkomplex und die letzte Nummer vor dem Abschluss bildete das erwähnte, vergleichsweise kurze Duett Fiorina/Masino. Darauf folgte bereits die Scena ultima mit neuem Rezitativ sowie einem vom Wiener Operndirektor Florian Leopold Gassmann komponierten Finalensemble „Non sei tu quella“. ${ }^{14}$ Insgesamt ergab sich für den dritten Akt eine Reduktion von 13 auf 6 Szenen, wobei vor dem Finale neben dem kleinen Duett lediglich mehrere aufeinanderfolgende kurze Ariosi der Livietta (Sängerin in

13 John Rice sieht das Vorhandensein eines Schlussensembles als wesentliches Differenzkriterium zwischen zweiteiligem Intermezzo und dreiaktigem Dramma giocoso. Vgl. John A. Rice, „The Roman Intermezzo and Sacchini's La contadina in corte“, in: Cambridge Opera Journal 12 (2000), S. 91-107, hier: S. 92 .

14 Die Wiener Fassung ist abgesehen vom Libretto durch drei vollständige Partituren dokumentiert (A-Wn, Mus. Hs. 1062, 1087 und 17826). In Mus. Hs. 17826 findet sich für das Finale (Bd. 3, fol. 21v-52r) wie für mehrere Einlagearien die Zuschreibung „Del Sig. ${ }^{r}$ Floriano Gas[s]mann“. 
Wien: Rosa Baglioni) sowie eine ebenfalls nicht besonders ausgedehnte Arie des Gianfriso (Giuseppe Pinetti) erhalten blieben. ${ }^{15}$

Die Bearbeitung brachte eine gewisse Veränderung der Personenhierarchie mit sich. Bei der Uraufführung wurde die Hauptintrigantin Auretta nochmals ausdrücklich herausgestellt, ${ }^{16}$ während der abschließende Coro, wie für eine solche Nummer üblich, alle Figuren der Handlung in musikalischer Einheit präsentierte. In Wien vollzog sich die Vereinigung aller drei Paare der Oper innerhalb des Finalensembles. Der Ensemblegedanke erscheint somit stärker betont, was bei einem Stück wie Le contadine bizzarre, das in besonders starker Weise von Einzelsituationen sowie ,Stimmungsbildern vom Lande lebt, einigen Sinn macht. Auf der anderen Seite bot das ausführlichere Schlussensemble die Chance, in einer Nummer gleich mehrere Figuren durch Solostellen zu exponieren, und dies wurde von Gassmann im Interesse seiner Stars, neben Pinetti vor allem Francesco Carattoli als Gianfriso sowie Teresa Eberardi als Auretta, genutzt. Eine gewisse Differenzierung im Ensemble bleibt also sichtbar, die Präsentation klarer Hauptfiguren erscheint jedoch in der Bearbeitung weniger deutlich.

Das auch für Wiener Verhältnisse drastische Bearbeitungsverfahren im dritten Akt von Le contadine bizzarre erklärt sich vor allem aus der Beschaffenheit der unmittelbaren Finalhandlung: In Venedig war dies eine komplizierte Geschichte um einen angeblichen Wunderbaum, aus dessen Wipfel man Dinge sehen soll, die in Wirklichkeit nicht geschehen. Dies führt zu ausgiebiger Kletterei mehrerer Personen, verbunden mit pantomimischen Darstellungen der am Fuße des Baums Stehenden, und damit zu einer nochmaligen kurzfristigen Verkomplizierung des Liebesreigens. Es kann nicht allzu sehr verwundern, dass diese reichlich abstrus wirkende und sich zudem in ausgiebigen Rezitativen entfaltende Handlung in Wien wenig Begeisterung erweckte. Dort bevorzugte man offenbar klarere dramatische Strukturen, weshalb die gesamte Handlung um den verzauberten Baum gestrichen und durch eine Gerichtsszene ersetzt wurde. Darin erscheinen fast alle Mitwirkenden als Angeklagte, während Gianfriso als Kläger und Richter in einer Person fungiert und eine Inhaftierung verfügt. Erst auf die durch laute Hilferufe aus dem Gefängnis unterstützte Nachricht, dass Auretta dort vor Angst und Schmerz zu sterben drohe, lässt er sich erweichen.

Das dem Ensemble vorangehende Rezitativ erscheint dabei kurz gehalten und führt lediglich in die Gerichtssituation ein, präsentiert juristisch gesprochen die Verfahrenseröffnung. Mit der Formulierung der Anklage beginnt das Ensemble und in der Folge setzt Gassmann die verschiedenen Stufen eines Gerichtsverfahrens in Musik: auf die Anklage folgen Vernehmung, Urteil, Bitten um Gnade, deren Ablehnung durch einen bereits unsicheren Gianfriso, Einkerkerung der Verurteilten und schließlich die überraschende Wende durch Aurettas vermeintlichen Zusammenbruch im Gefängnis. Ein Ensemble also, das verschiedene Affekte in sich vereinigt, dessen Handlung hinreichend Anregungen für musikalische Formgestaltung bietet und für das man bereits den Terminus „Aktionsfinale“ verwenden kann.

Bezeichnend für die Gerichtssituation mit den unruhigen Angeklagten auf der einen und dem um richterliche Würde bemühten Gianfriso auf der anderen Seite erscheint die

15 Mit dem dritten Akt herrschte offenbar häufiger Unzufriedenheit, einige Änderungen finden sich etwa auch in den Libretti zu den Aufführungen Lucca 1766 sowie Mailand 1766. Giuseppe Pinetti sang schon bei der Uraufführung, seine Arien blieben in Wien unverändert.

16 Außer ihrem Duett mit Nardone unmittelbar vor dem Finale hatte sie im dritten Akt eine Cavatina zu singen. Beide Nummern entfielen in der Wiener Bearbeitung. 
Tempobezeichnung „Allegro maestoso“ des ersten Teils (G-Dur, C). Die diesen Abschnitt abschließende Bekräftigung des Urteils (,io comando, e così va“) lässt Gassmann gleichzeitig mit dem Protest Nardones („questa è troppa crudeltà") singen und ermöglicht so ein direktes musikalisches Aufeinandertreffen der Stimmen von Pinettis Buffotenor und Carattolis Bass. ${ }^{17}$ Der Tenor fungiert hier als lächerlich wirkende Amtsperson, er steht am Schluss als Verlierer und als einziger ohne Braut da, während die Bassrolle, der „contadino ricco e sciocco" Nardone, auch Züge des Liebhabers trägt.

Allegro maestoso

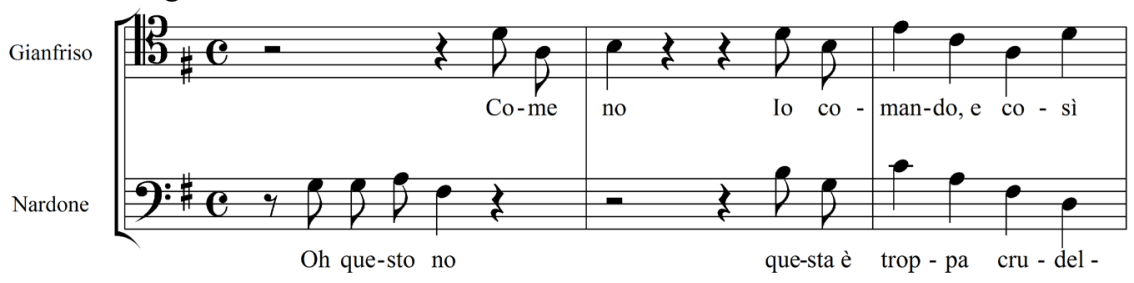

G.

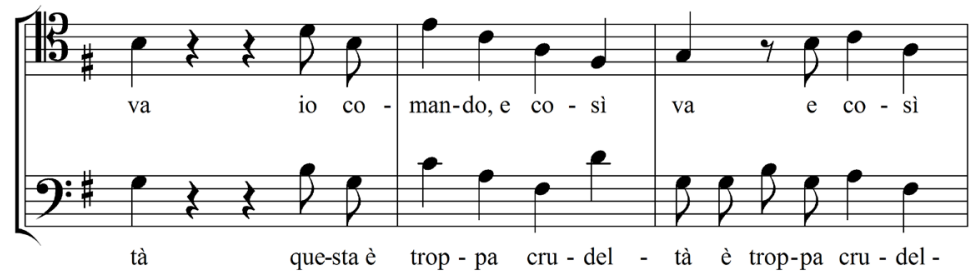

G.

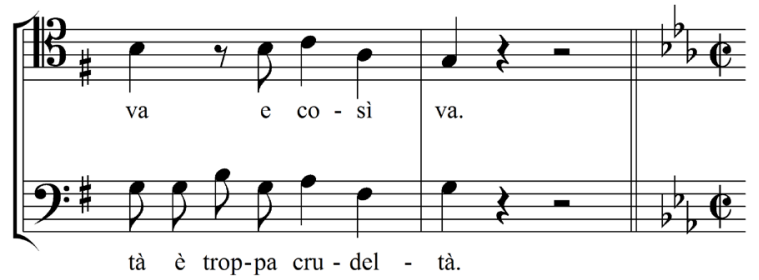

Notenbeispiel 1: Le contadine bizzarre, Finale III. Akt, A-Wn, Mus. Hs. 17826, Bd. 3, fol. $27 \mathrm{r}-28 \mathrm{r}$

Mit den Gnadenbitten setzt der zweite Finalteil an (Larghetto, Es-Dur, $\boldsymbol{\oplus}$, Beginn im Piano), in dem zunächst Auretta durch eine Solostelle herausgehoben erscheint und anschließend Gianfriso versucht, bedrohlich und unerschütterlich zu wirken („Son più duro d'un Nerone"), was allerdings musikalisch nicht ganz überzeugend gerät.

17 Für Carattoli vertonte Gassmann auch die Arie im ersten Akt „Quest'aria non è nobile“ neu, eine weitere Arie Nardones im ersten Akt wurde ersetzt. 

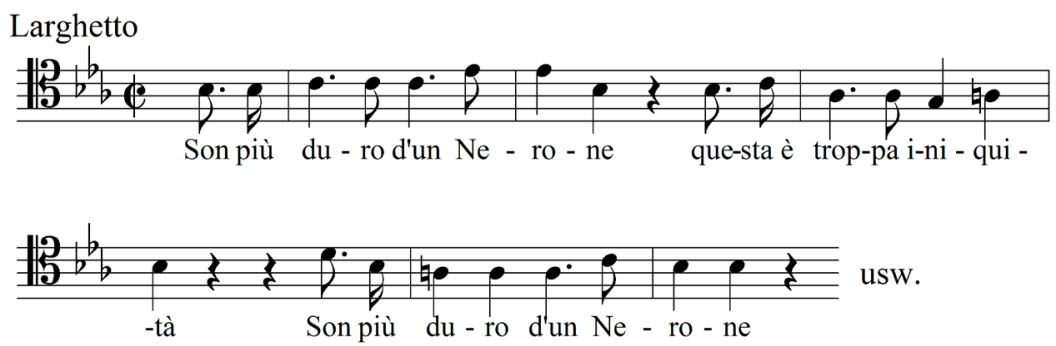

Notenbeispiel 2: Le contadine bizzarre, Finale III. Akt, A-Wn, Mus. Hs. 17826, Bd. 3, fol. 30r-v

Noch innerhalb des Larghetto schafft Gassmann verstärkte Unruhe in Form einiger rasch abwechselnder Kurzsätze Aurettas (verschiedene Bitten) und Gianfrisos (abwehrende Gesten wie „non ascolto“ oder „in prigion“), gefolgt durch ausführlichere, von falscher Pathetik geprägte nochmalige Beschwerden Aurettas über die Ungerechtigkeit des Gerichts. Daran schließen sich Parlando-Kommentare Nardones an, zwischen gespieltem Mitleid und unterdrücktem Lachen, während Auretta und ihre vermeintlichen Komplizen abgeführt werden. Auf engem Raum, innerhalb eines Teils des Finalensembles, finden sich somit eine Fülle unterschiedlicher Emotionen und Reaktionen von Flehen über Schimpfen und Wut bis hin zu (falschem) Pathos und Mitleid.

Im anschließenden Abschnitt (Wechsel zum 3/4-Takt) bildet die Intervention von Fiorina und Masino, die mit Auretta eingesperrt wurden und nun gemeinsam um Hilfe rufen, einen finaltypischen, allgemeine Konfusion provozierenden Colpo di scena. Nach schließlich erreichter Begnadigung inklusive der Feststellung, dass Gianfriso gehörnt wurde, erfolgt ein nochmaliger Wechsel zum 6/8-Takt. In diesem vorletzten Abschnitt des Finales bilden sich musikalische Paare, wobei sich allerdings Nardone überraschend dem „Ah villanacci, ah mascalzoni ladri bricconi“ Gianfrisos anschließt, während Auretta zusammen mit den an diesem Finale teilnehmenden Parti serie Rosalba und Lucio notiert ist. Das Tempo lautet nach wie vor Larghetto, ein Wechsel zum Presto erfolgt erst unmittelbar vor dem von allen Figuren gesungenen Schlussmotto, also angesichts der turbulenten Handlung eigentlich überraschend spät. Gassmann bevorzugt zunächst andere Parameter wie etwa rasche Achtelketten in den Singstimmen oder Crescendi, um mehr Bewegung zu erzeugen, und spart das schnelle Tempo für den endgültigen Schluss auf.

Im abschließenden Tutti wird Gianfriso zu Beginn noch von den anderen Stimmen abgesetzt und singt als einziger die ersten beiden Zeilen nicht mit („A gustare ognun s'appresti / Dell'amore i dolci frutti.“). Stattdessen erhält er mit „Io a restare con denti asciutti / Io son solo, e bene mi sta“ nochmals eine Solophrase. Von der Liebe bleibt er also ausgeschlossen (nicht ohne Erleichterung), kann sich jedoch der abschließenden Erkenntnis, genarrt worden zu sein, nicht verschließen und stimmt somit in die Schlussworte „Ecco fatto il becco all'Oca, / E le Corna al Podestà “ mit ein. Die sich durch eine Ensemblenummer ergebende größere Flexibilität im Zusammenspiel der Personen, also die Möglichkeit, ganz unterschiedliche Figuren herauszuheben oder zusammenzuspannen (Auretta versus Gianfriso, Gianfriso versus Nardone ${ }^{18}$ usw.) scheint ein wesentliches Anliegen bei der Gestaltung des neuen Finalensembles gewesen zu ein.

18 Gerade die Interaktion der beiden Männer Carattoli und Pinetti (beide mit Sicherheit talentierte Darsteller) scheint Gassmann gereizt zu haben. Bei einem traditionell gestalteten Finalkomplex mit einem 
In der bereits in Bezug auf das erste Finale erwähnten Oper La conversazione von Giuseppe Scolari kam es wie in Le contadine bizzarre nicht nur zur Abänderung des eigentlichen Finales, sondern zu einer Modifikation des gesamten Schlussaktes in struktureller, inhaltlicher wie musikalischer Hinsicht. Die Anzahl der Solonummern wurde erweitert mit zwei zusätzlich eingefügten Arien für Don Fabio (Giuseppe Andreoli) in der ersten sowie für Madama Lindora (Francesca Buini) in der zweiten Szene. ${ }^{19}$ Ab der dritten Szene ergaben sich inhaltliche Veränderungen mit Auswirkungen auf die gesamte finale Szenenfolge. In der Uraufführungsversion beschließt Lucrezia, nachdem sie erfahren hat, dass Sandrino ${ }^{20}$ und Don Fabio zu einem geplanten Fest maskiert kommen wollen, sich ebenfalls zu verkleiden, und zwar als Türkin. Dies gibt Anlass zu der Arie „Salamelecch / stara Sultana / con Ottomana“, die ihren Witz wie unschwer zu erkennen aus einem vermeintlich ,türkischen“ Tonfall zieht. Die beiden Herren erscheinen hingegen in der vierten Szene „vestiti da calabresi“, in der Hand das in Süditalien verbreitete Instrument Calascione, was Anlass zu einem im Dialekt gesungenen Lied („La carciofol““) bietet. Diese heitere Stimmung wird in dem anschließenden Duett von Madama Lindora und Giacinto aufgenommen, die gewissermaßen tanzend zusammenfinden. ${ }^{21}$

Von all dem blieb in Wien kaum etwas erhalten, abgesehen von dem kalabresischen Lied, bei dem jedoch statt Sandrino Giacinto sang, der damit eine recht bescheidene Kompensation für sein gestrichenes Duett mit Madama Lindora erhielt. Die türkische Maskerade der Lucrezia, nach der Arie „Salamelecch“ ohnehin im Libretto nicht weiter thematisiert und damit lediglich ein für die Handlung eigentlich überflüssiger Farbtupfer, entfiel. Stattdessen fanden in Wien Lucrezia und Sandrino zusammen, allerdings nicht in einem Duett, sondern in zwei aufeinanderfolgenden Einzelnummern, für Sandrino die Instrumentenarie "S'ha da ballare, s'ha da cantare“, entnommen aus Domenico Fischiettis Il mercato di Malmantile, ${ }^{22}$ für Lucrezia eine Kanzone unbekannter Herkunft im neapolitanischen Dialekt, „Fegliole semplecelle“, als Ersatz für „Salamelecch“. ${ }^{23}$ Damit glich sich ihre Solonummer der Stimmung des darauf folgenden kalabresischen Gesangs an, der ebenfalls im 6/8-Takt steht und nur von Streichern begleitet wird. Auf die ursprünglich vorhandene Varietas, negativ formuliert: auf den willkürlich anmutenden Kontrast von süditalienischem Dialektlied und „alla turca“, wurde verzichtet.

Duett, üblicherweise ein Liebesduett für Mann und Frau, und dem einheitlichen Coro finale, hätte diese Möglichkeit kaum bestanden.

19 „Non temo d'un esercito” (komponiert von Niccolò Piccinni ursprünglich für Le vicende della sorte, Rom 1761) wurde von Andreoli in Wien auch in La vedova ingegnosa verwendet, es handelte sich also um eine typische ,Kofferarie‘. Ursprünglich entstand die Rolle des Don Fabio in Venedig für Francesco Carattoli. Die Herkunft der Sopranarie „Bel vedere in allegria“ konnte bislang nicht geklärt werden.

20 Giacomo Tibaldi, ursprünglich eine für Francesco Baglioni geschriebene Rolle.

21 Die Szenenanweisung lautet „Si suona il minuetto e fanno la riverenza“. Eine der Uraufführung entsprechende Partitur scheint nicht zu existieren. Das Manuskript in I-Fc, B. I. 647-649, neben A-Wn, Mus. Hs. 18042 offenbar das einzig überlieferte Gesamtmanuskript, enthält zwar den Coro finale der Uraufführung und steht dieser insgesamt näher als die Wiener Version, das letzte Duett ist dort jedoch durch ein anderes ersetzt.

22 Bei den Wiener Aufführungen dieser Oper 1763, also im selben Jahr wie La conversazione, wurde die Arie durch eine andere Nummer ersetzt.

23 In italienischen Dialekten gehaltene Librettoabschnitte wurden bei Wiener Aufführungen häufig, aber keineswegs durchgängig eliminiert, wie das Beispiel von La conversazione zeigt. In Einzelfällen wurde auch statt eines hochitalienischen Textes eine Dialektarie eingefügt (etwa für Francesco Carattoli). 
In Wien kam es laut Libretto vor dem „alla calabrese“ zu einem Szenenwechsel („Sala illuminata, con sedie. Madama, poi Giacinto, e Sandrino mascherati“24) und auf diese Nummer folgte die letzte Szene mit Rezitativ sowie dem abschließenden kurzen Coro finale „Libertà, libertà“, der die analoge Nummer „E qui la nostra conversazione“ ersetzte. Bei der Uraufführung hingegen fand der Szenenwechsel erst unmittelbar vor der durch einen Instrumentalsatz eingeleiteten Scena ultima statt und die Anweisung lautete abweichend „Sala illuminato [sic] per la Festa de Ballo. / Tutti / Si fanno varj Minuetti, ed altri Balli a piacere dopo di che si termina col seguente Coro" ${ }^{25}$. Die Oper schloss also in Venedig mit einer durch den Inhalt des (in Wien fehlenden) Liebesduetts vor dem Finale schon vorbereiteten Tanzszenerie. Im Wiener Libretto findet sich zwar nach dem kalabresischen Lied ebenfalls eine Szenenanweisung „si intrecciano vari minuetti“, in der Partitur jedoch fehlt eine separate Instrumentalmusik, und so erscheint das Tanzelement, in Venedig prägend für die gesamte finale Nummernfolge, zurückgedrängt. Wie bei Le contadine bizzarre werden pantomimische Elemente, die für die Handlung kaum erforderlich sind, sondern ein reines Spiel um des Spiels willen darstellen, reduziert.

\section{Opera buffa und Vaudeville-Finali}

Die Bearbeitung der 1767 und 1770 in Wien gespielten La contadina in corte von Antonio Sacchini $^{26}$ zeigt ebenfalls, dass eine allzu verwickelte, ungewöhnliche inhaltliche Elemente bietende Finalhandlung Missfallen erwecken konnte. Bei der Uraufführung in Rom ${ }^{27}$ bot das obligatorische Duett vor dem Finale statt der Vereinigung des Liebespaares eine Liebesprobe: Tancia verkleidet sich als Mann und versucht, Sandrina zu verführen, die jedoch widersteht und so ihre Treue zu Ruggiero beweist. Der Reiz dieses Duetts (in Rom gesungen von zwei Kastraten in Frauenrollen) bestand nicht nur in dem gewissermaßen potenzierten Spiel der Geschlechter, sondern auch in der Musik, die so interpretiert werden kann, als seien durchaus echte Gefühle bei Sandrina wie Tancia im Spiel.

In Wien, wie auch an anderen Orten vornehmlich im deutschsprachigen Raum, ging dies wohl zu weit und es kam zu einer Ersetzung des Duetts. Für die Wiener Aufführung komponierte abermals Gassmann eine neue Nummer, „Mi permetta per favore“, 28 in der Ruggiero selbst sich verkleidet und seine Geliebte auf die Probe stellt. Tancia fungiert le-

24 Libretto zur Wiener Aufführung von La conversazione, 1763, S. 41 (A-Wn, 641432-A.11,1 Mus).

25 Zitiert nach dem Libretto zur Uraufführung in I-Mb, S. 46. <http://www.braidense.it/rd/00414.pdf>, 9.1.2012).

26 Vgl. auch Rice, „The Roman Intermezzo“.

27 Es existieren zwei weitgehend übereinstimmende Libretti für 1765 und 1766, die aber unterschiedliche Sängernamen nennen, und zwar das jeweils am Teatro Valle aktive Ensemble. Ob hier der ungewöhnliche Fall einer Wiederaufnahme gleich im Jahr nach der Uraufführung vorliegt oder die Aufführung 1765 nicht stattfand, ist bis heute nicht definitiv geklärt.

28 Enthalten auch in den Partituren zu La contadina in corte in A-Wgm, IV 15747 / Q 1973; I-Nc, 31.4.20-21; I-MOe, Mus. F. 1033 sowie I-Bc, KK.18, alles Wiener Abschriften, die die Fassung von 1767 wiedergeben, deren Änderungen bei der Wiederaufnahme 1770 zum Teil übernommen wurden. Auf einer Wiener Partitur basierte offensichtlich auch die Aufführung in Regensburg (o. J., konsultiertes Libretto D-Mbs, P.o. it. 292 m). Libretto wie Klavierauszug und Stimmenmaterial in D-Rtt, Sacchini 7/I-II (neue Signatur: 424-425) enthalten ebenfalls mehrere der Wiener Einlagen, darunter wiederum Gassmanns Duett. Ebenso beachtlich wie die (in dieser Intensität ungewohnte) Diffusion von Wiener Partituren zu La contadina in corte ist die Anzahl von sicher oder wahrscheinlich aus römischen Kopistenwerkstätten stammenden Exemplaren (I-Rdp, 123/A; I-Rc, Fondo Baini Mss. 2824-2825; I-Fc, 
diglich als Zeugin, und so erscheinen sämtliche möglichen Implikationen von gleichgeschlechtlicher Liebe ausgeschlossen. Der Coro finale von La contadina in corte, „Semplice io nacqui“, blieb erhalten, jedoch wurde bei der Wiener Wiederaufnahme von 1770 ein neuer Teil in Form eines Vaudeville-Finales daran angehängt. ${ }^{29}$

Die Änderungen stehen in Zusammenhang mit einer allgemeinen Glättung des römischen Librettos, das auch sozialkritische Aspekte aufscheinen lässt. Im Gegensatz zu vielen anderen Opere buffe endet La contadina in corte wirklich mit der Heirat eines aus unterschiedlichen Schichten stammenden Paares, dem Baron Ruggiero und der Bäuerin Sandrina, die sich am Schluss nicht etwa als adelig entpuppt wie zum Beispiel Cecchina. In Rom wies Sandrina zudem eine gewisse Ambivalenz auf und artikulierte ihre Motivation, durch Heirat der Armut entfliehen zu können. Auch Berto, den Sandrina zu Beginn aufrichtig liebt, zeigt sich aufsässig und setzt der naiven Naturschwärmerei des Barons den trockenen Hinweis auf die Armut der Landbevölkerung entgegen. ${ }^{30}$ In Wien verfolgten die Bearbeiter hingegen die Prämisse, dass Sandrina wenigstens über einen moralisch einwandfreien Charakter verfügen müsse, um so die Heirat mit Ruggiero (der hier auch Bedenken gegen die nicht standesgemäße Verbindung äußert) zu rechtfertigen. Gerade aus diesen Gründen wies die Wiener Version von La contadina in corte zwar nur einen durchschnittlichen Anteil an ersetzten Arien auf, jedoch einige intensiv bearbeitete Rezitative. ${ }^{31}$ Letztlich, und dies ließe sich auch für Le contadine bizzarre sagen, boten die neuen Finalnummern im Vergleich zur bunten Handlung der Uraufführung textlich konventionellere Lösungen.

In Gli uccellatori, der einzigen in diesem Zeitraum aufgeführten Opera buffa Gassmanns, die er nicht direkt für Wien komponierte, kam es gegenüber der venezianischen Uraufführung ebenfalls zu einer Ersetzung des Duetts vor dem Finale (Roccolina/Cecco), allerdings ohne dramaturgische Konsequenzen. ${ }^{32}$ Auf das Duett folgte in Wien direkt ein neues Finalensemble, „Si cara, vi credo“, das sowohl das letzte Rezitativ als auch den sechszeiligen Coro finale „Quel bambinel d'amore“ ersetzte. Das Zusammenfinden der beiden anderen Liebespaare Mariannina/Toniolo sowie Contessa Armelinda/Marchese Riccardo vollzog sich damit innerhalb des Ensembles und nicht mehr wie bei der Uraufführung im Rezitativ. ${ }^{33}$ Beide Parti serie sind hier also abermals an einem wirklichen Finalensemble und nicht mehr lediglich an einem vom Solistentutti gesungenen Abschlusschor beteiligt.

Auch Galuppis Il villano geloso zeigt eine Neugestaltung des Schlussfinales und einmal mehr findet sich ein Gassmann zugeschriebenes Ensemble, das Liebesduett, Coro finale

D.I. 639-640; P-La, 46-III-35 e 36; D-Wa, 46 Alt 121-122 und vielleicht B-Bc, 2333, die vermutlich auch für eine Aufführung im französischen Sprachraum verwendet wurde).

29 Dokumentiert nur über das Wiener Libretto von 1770. Das neue Duett Gassmanns wurde auch 1770 gespielt.

30 Szene I,2: „Signor di questi Colli / ne trovarete mille / Più leggiadri, e più ameni: quivi solo / Regna la Povertà.". Libretto zu La contadina in corte, Rom, Teatro Valle, 1765, S. 6. Konsultiertes Libretto: B-Bc, 19.729.

31 A-Wn, Mus. Hs. 17815 zeigt gerade bei den Rezitativen zahlreiche Bearbeitungsspuren (Überklebungen, Ausstreichungen, Korrekturen). Einige Rezitative existieren doppelt, sodass nur durch Vergleiche mit dem Libretto sowie den ,sauberen' Kopien der Wiener Partiturfassung erschlossen werden kann, was letztlich gesungen wurde. Auffallend ist der Anteil der komplett neu gefassten Rezitative.

32 A-Wn, Mus. Hs. 18080 kann der Wiener Aufführung zugeordnet werden, P-La, 44-VII-46 a 48 der Uraufführung, I-Fc, D.I.237-239 einer Bearbeitung für Florenz 1761.

33 Ein ähnliches Vorgehen zeigt La serva astuta, wozu bislang keine Partitur bekannt ist. Dort steht nach dem Rezitativ lediglich ein vierzeiliger Schlusschor. In Wien setzte nach einem Teil des ursprünglichen Rezitativs ein neu eingefügtes Ensemble ein, in dessen Rahmen die Handlungsauflösung stattfand. 
sowie das dazwischenliegende Rezitativ ersetzt. ${ }^{34}$ Gassmanns Finale beginnt mit einem Duettino für Giannina und Pierotto, als dessen Text er, in verkürzter Form, den des ursprünglichen Liebesduetts verwandte. Aus dem großen Duett vor dem Coro finale, einer unabhängigen, durch ein Rezitativ klar abgesetzten Musiknummer, wurde somit der Einleitungsteil eines Finalensembles.

Die neue Schlussnummer von Il villano geloso mündet in ein Vaudeville-Finale und diese Form scheint sich in Wien einiger Beliebtheit erfreut zu haben. Ein eingelegtes Vaudeville findet sich nicht nur in Il villano geloso, sondern wie erwähnt auch in La contadina in corte sowie in Piccinnis Lo sposo burlato. Während es sich bei Il villano geloso jedoch insgesamt um ein neues Finalensemble handelt, bleibt in den anderen genannten Opern, beides Importe aus Rom, das ursprüngliche Ensemble bestehen und ein Vaudeville-Teil wird daran angehängt bzw. eingebaut.

Wie dies musikalisch funktioniert, zeigt Lo sposo burlato, geschrieben 1769 für das Teatro Valle in Rom und ein Jahr später nach Wien gelangt, eventuell durch Vermittlung von Francesco Bussani, der bei der Uraufführung mitgewirkt hatte. Insgesamt gehört dieses Werk zu den weniger bearbeiteten Opern. Lediglich eine Sopranarie wurde in Wien zusätzlich eingefügt, drei weitere Arien neu vertont. Alle drei Neuvertonungen betrafen die Partie der Lindora, die in Rom von einem Kastraten (Pietro Benedetti) gesungen worden war, dessen gesangliche Fähigkeiten die der unbekannten Wiener Primadonna ${ }^{35}$ überstiegen haben müssen und der über einen deutlich größeren Stimmumfang verfügte. In den Ensembles erscheint die Rolle der Lindora wie in den Arien hinsichtlich der Verzierungen vereinfacht und alle $f$ " übersteigenden Töne wurden nach unten transponiert.

Die nötigen Modifikationen bei der Gesangspartie der Lindora bieten allerdings keine Erklärung dafür, dass dem Schlussfinale, in diesem Fall das Ende des zweiten Akts, ein neuer Teil hinzugefügt wurde. Im Libretto der Uraufführung und im Partiturmanuskript ${ }^{36}$ findet sich am Ende das Finalensemble, „Belle luci di diamante“: Don Pomponio erfährt, dass er genarrt wurde und die vermeintliche „Hochzeitsprobe“, bei der Florindo die Rolle des Bräutigams spielte, in Wirklichkeit eine gültige Vermählung war. Die verschiedenen Intrigenfäden entspinnen sich, Don Pomponio wütet zunächst, lässt sich aber durch die Bitten der anderen Figuren erweichen und fügt sich in sein Los.

Bei der Uraufführung endete an dieser Stelle das Finalensemble mit einem konventionellen harmonischen Schlusstutti „S'onori un tal giorno“. Im Wiener Libretto hingegen folgt auf diesen Abschluss noch ein Vaudeville, eingeleitet durch eine Solostrophe des Don Pomponio, an die der Refrain anschließt: „E le donne in conclusione / sembran nate a comandar". Danach geben die übrigen drei Figuren ihr musikalisches Statement ab, immer gefolgt vom Refrain, der zunächst am jeweiligen Strophenende solo intoniert und dann von allen vier Sängern gemeinsam wiederholt wird. Mit dieser „Moral von der Geschichte“ anstelle eines Jubelchors endet die Oper im Wiener Libretto.

Das Partiturmanuskript der Wiener Fassung ${ }^{37}$ zeigt jedoch, dass hier wohl ein Fehler des Librettodruckers vorliegt. Der neue Vaudeville-Teil erscheint in der Partitur einge-

$34 \mathrm{Zu}$ dieser Oper scheint nur die Partitur A-Wn, Mus. Hs. 18073 zu existieren, welche die Wiener Fassung wiedergibt und in der die Namen der Komponisten zahlreicher Einlagearien notiert sind.

35 In den Wiener Libretti fehlen 1767 teilweise und in den Jahren ab 1768 generell die Besetzungen.

36 I-Nc, 16.3.29-30; <http://www.internetculturale.it/opencms/opencms/it/viewItemMag.jsp?case=\&id= oai\%3Awww.internetculturale.sbn.it\%2FTeca $\% 3$ A20\%3ANT0000\%3AIT\%5C\%5CICCU\%5C\%5 CMSM\%5C\%5C0152783>, 9.1.2012.

37 A-Wn, Mus. Hs. 17816 (das Finale in Bd. 4, fol. 54v-105r). 
schoben hinter einem Ensembleteil (Allegro, 6/8-Takt) und vor dem abschließenden Tutti „S'onori un tal giorno“. Außerdem beginnt dieser Abschnitt mit dem von allen Figuren gesungenen Refrain, dann erst folgt die erste Solostrophe von Don Pomponio sowie die weiteren drei Strophen, alle jeweils abgeschlossen durch den Refrain, zuerst im Solo und dann wiederholt im Tutti. Lediglich nach der letzten Strophe, intoniert von der Intrigantin Livietta, fehlt die von allen gesungene Refrain-Wiederholung. Stattdessen folgt eine Generalpause mit anschließendem Taktwechsel zum 3/8-Takt und das Tempo wechselt zum Allegro spiritoso, womit auch das Wiener Finale in den ursprünglichen Finalchor „S’onori un tal giorno" mündet, der um ein paar Takte ausgedehnt erscheint. Die offenkundige Vorliebe für Vaudeville-Finali legt eine auf einen spezifischen, Wiener Geschmack' ausgerichtete Bearbeitung und Verbindungen zum sonstigen damaligen Repertoire der Wiener Bühnen nahe, insbesondere zur intensiven Pflege der französischen Oper. ${ }^{38}$

\section{Neue Finalmusik zu altem Text}

Einen von den bislang behandelten Beispielen abweichenden Fall präsentiert La cascina von Giuseppe Scolari, für die in der Wiener Nationalbibliothek sowohl eine Abschrift der Wiener Aufführungspartitur als auch die aus Venedig importierte Quellenpartitur existieren und zudem eine Sammlung von einigen durch Gassmann auf die alten Texte neuvertonten Einzelnummern. ${ }^{39}$ Scolaris Oper wurde 1756 am Teatro San Samuele in Venedig uraufgeführt und in der Folge bis in die 1770er Jahre hinein an zahlreichen Theatern nachgespielt. ${ }^{40}$ Erst zwölf Jahre nach der Uraufführung gelangte das Stück nach Wien (1768). Das dortige Libretto nennt zwar noch den Textdichter Goldoni, nicht jedoch den Komponisten Scolari. Stattdessen findet sich die Angabe „La Musica è di diversi autori“, und in der Tat handelt es sich um eine besonders intensive Bearbeitung, bei der nur weniger als ein Drittel der Nummern erhalten blieb. Gassmann war im Fall von La cascina wie generell in diesen Jahren der Hauptbearbeiter und griff nachweislich gerade bei den Ensembles ein, erklärte sie gewissermaßen zur ,Chefsache ${ }^{6}$ des Operndirektors. ${ }^{41}$

Von drei Aktfinali blieb lediglich das zum ersten Akt unangetastet. Im dritten Akt wurde das letzte Rezitativ gestrichen und ging nun inhaltlich im ersten Teil eines umfangreicheren Schlussensembles auf. Das Finale folgte damit ohne Unterbrechung durch ein Rezitativ direkt auf das Duett des Hauptliebespaares, bei dem es sich ebenfalls um eine

38 In dieser Hinsicht äußerte sich bereits Schneider, der auch die wechselseitige Beeinflussung von Buffa und Opéra comique bei der Finalgestaltung betonte und speziell hinsichtlich des Vaudeville-Schlusses zudem Glucks Orfeo als Vorbild nannte. Vgl. ebenfalls John Rice, Antonio Salieri and Viennese Opera, Chicago und London 1998, S. 109.

39 Wiener Partitur: A-Wn, Mus. Hs. 1065; Venezianische Quellenpartitur für die Wiener Aufführung (mit vereinzelten Eintragungen für die Bearbeitung): A-Wn, Mus. Hs. 17852; Einlagenummern Gassmanns: A-Wn, Mus. Hs. 3979 (und Mus. Hs. 3980 mit der neu eingefügten Arie für Berto aus II, 10, „Pippo, consolati").

40 Vgl. Claudio Sartori, I libretti italiani a stampa dalle origini al 1800, Bd. 2, Cuneo 1990, S. 78-80.

41 Das neukomponierte Finale ist in dem erwähnten Band mit den von Gassmann neuvertonten Nummern in A-Wn, Mus. Hs. 3979 enthalten (fol. 31r-53v), in der Wiener Gesamtpartitur A-Wn, Mus. Hs. 1065 in Bd. 2, fol. 117r-135v, und das Finale der Uraufführung von Giuseppe Scolari in A-Wn, Mus. Hs. 17852, Bd. 2, fol. 65r-84r. 
Einlage handelte. ${ }^{42}$ Die Auflösung der Handlung erscheint also auch in diesem Beispiel ,musikalisiert'.

Den Abschlussteil des Finalensembles im dritten Akt bildete der Text des Coro finale der Uraufführung „Sposi già siamo“. Ob hier auch die Musik Scolaris erhalten blieb, also eine Erweiterung der ursprünglich schon vorhandenen Schlussnummer stattfand wie bei Lo sposo burlato, ist nicht verlässlich zu beurteilen, da wegen des Fehlens des kompletten letzten Aktes in der Partitur zur Wiener Aufführung die Veränderungen nur aus dem Libretto erschlossen werden können.

In Libretto und Partitur überliefert ist hingegen die Wiener Version des Finales zum zweiten Akt. Gassmann entschloss sich hier zu einer Neuvertonung des alten Textes unter Rückgriff auf die ursprüngliche musikalische Fassung des Kollegen. Seine Partitur weist im Vergleich zu Scolari drei zusätzliche Stimmen auf, zwei Oboen und ein Fagott, außerdem erscheinen die Bratschen separat notiert und führen auch ein musikalisches Eigenleben. Beide Finali stehen in F-Dur, beginnen im 2/4-Takt (Allegretto bei Scolari, Andantino bei Gassmann) auftaktig mit einer punktierten Sechzehntelnote in den Violinen und verwenden zu Beginn Triolen.

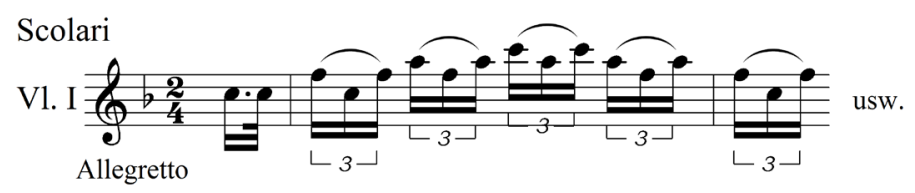

Gassmann

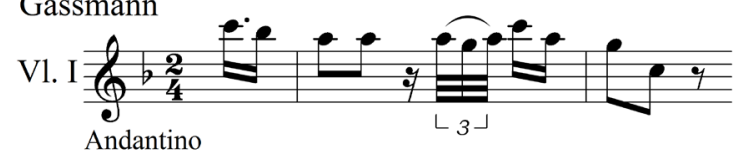

Notenbeispiel 3: La cascina, Finale II. Akt, A-Wn, Mus. Hs. Bd, 2, fol. 65r (Scolari) und A-Wn, Mus. Hs. 1065, Bd. 2, fol. 117r (Gassmann)

Während jedoch in der ursprünglichen Version die Violinen gleichmäßige Triolenketten spielen und in den anderen Stimmen durch jeweils eine Achtelnote auf der ersten Taktzeit begleitet werden, erscheinen in der Neuvertonung Rhythmus und Begleitung flexibler und ausdifferenzierter, etwa in der Basslinie mit ihren Oktavsprüngen und dem synkopierten Rhythmus.

Auf den ersten Blick ist zu erkennen, dass Gassmann deutlich mehr Wert auf die Instrumentation legt, und der Wunsch, eine anspruchsvollere Orchesterbegleitung zu schaffen, bei der die Instrumente im Vergleich zum Finale Scolaris individueller hervortreten, dürfte ein wichtiger Grund für den Entschluss zur Neuvertonung gewesen sein. Auch mit der Behandlung der Singstimmen, die bei Gassmann teilweise mehr verziert und ebenfalls rhythmisch wie harmonisch ausgefeilter erscheinen, herrschte vermutlich Unzufriedenheit.

Schon die ersten Takte des Finales zeigen jedoch auch Gassmanns Anklänge an Scolari, die sich im weiteren Verlauf der Nummer auf unterschiedliche Weise fortsetzen. Zum einen hielt sich der Wiener Kapellmeister zunächst an die schon bei Scolari existierende Aufteilung des Ensembles, bei dem die einzelnen Abschnitte recht kurz sind.

42 „Ah tu mi lasci, oh cara“ statt dem ursprünglichen „Pastorelli, io son da vendere“. Vom Text her passt das spielerische Duett der Uraufführung eigentlich besser zu den beiden Figuren Pippo und Lena als das vom sprachlichen Duktus her im Seria-Stil gehaltene Einlageduett. 
Den Colpo di scena dieses zweiten Finales bildet das Erscheinen des als Frau verkleideten Berto bei der Textstelle „Pippo caro caro caro“.43 Beide Vertonungen (Scolari etwas früher) wechseln hier zu einem langsameren Tempo und zum 6/8-Takt, was sich aus dem Text heraus ergibt, da ein solistischer auf einen chorischen Teil folgt und im Metrum Ottonari die vorherigen Quinari ablösen. Die Instrumentalbegleitung dieses Abschnittes erscheint in beiden Vertonungen unterschiedlich, die Melodie der Singstimme hingegen ist eine wörtliche Übernahme von Musik Scolaris durch Gassmann. Nach drei Takten allerdings beginnt die Gesangslinie gegenüber Scolari zu variieren, um sich schließlich im weiteren Verlauf anders fortzusetzen.

Scolari
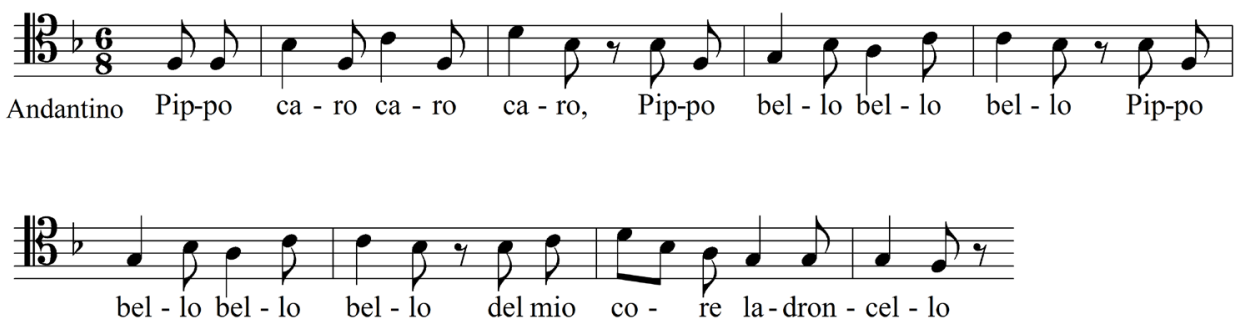

Gassmann
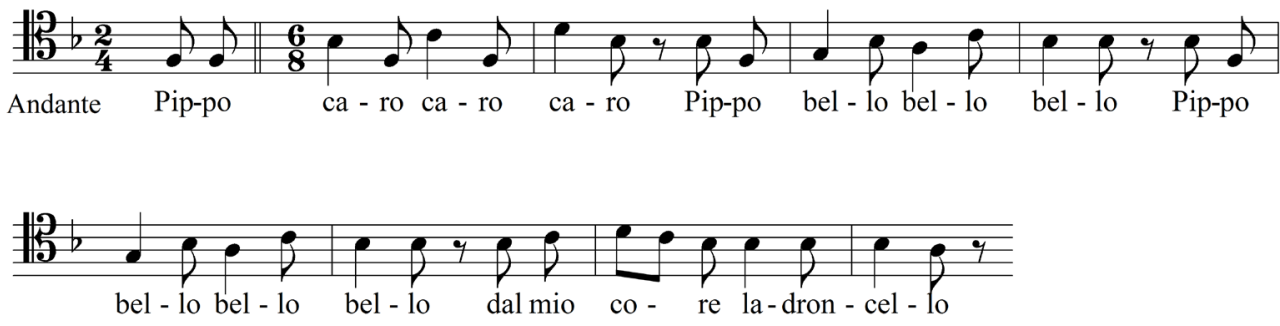

Notenbeispiel 4: La cascina, Finale II. Akt, A-Wn, Mus. Hs. 17852, Bd. 2, fol. 71v-72r (Scolari) und A-Wn, Mus. Hs. 1065, Bd. 2, fol. 125r-125v (Gassmann)

Die letzten drei Noten der Singstimme sind bei Gassmann wieder identisch mit Scolari und beide vollziehen einen erneuten Tempo- und Taktwechsel exakt an derselben Stelle, nämlich vor der letzten Silbe / Note der Singstimme bei „pie-tà“. Beide wählen für den hier beginnenden Abschnitt den 3/4-Takt und ein schnelles Tempo, Scolari Presto, Gassmann Allegro. Musikalische Übernahmen in der Singstimme jedoch kommen nicht mehr vor und generell trennen sich die Wege der Komponisten nun stärker. Vor Beginn des „a 5“ und „Oh che sorpresa è questa“ kehrt Scolari zum 6/8-Takt zurück, während Gassmann lediglich durch eine Generalpause absetzt. Der abschließende schnelle Teil (Allegro assai bei Scolari, Presto mit gleichzeitigem Wechsel zum $\mathbf{C}$-Takt bei Gassmann) beginnt in beiden Vertonungen an jeweils unterschiedlichen Textstellen.

In solchen, durch die Solisten chorisch gesungenen Abschnitten zeigen sich generelle Unterschiede zwischen den Komponisten: So lässt Scolari von allen intonierte Phrasen gerne im Parlando in schnellen, gleichmäßigen Achtelnoten ausklingen, eine Konvention, die

43 Im Wiener Libretto „Pippo caro, Pippo bello“. 
Gassmann meidet. Der Wiener Kapellmeister hingegen bricht einen Chorsatz häufiger auf, um einzelne Figuren oder Paare musikalisch und/oder textlich hervortreten zu lassen. So gestaltet etwa Scolari seinen Schlussabschnitt ab „è una cosa portentosa“ fast vollkommen syllabisch und als kompakten homophonen Chor-Block, obwohl zwei abweichende Texte gesungen werden.

Gassmann, der seinen Schlussteil etwas früher beginnen lässt, setzt die einzelnen Figurengruppen zunächst voneinander ab und verdichtet den Satz erst allmählich immer mehr, um die Singstimmen in den allerletzten Takten zu musikalischer Einheit zusammenfinden zu lassen. Damit zeigen sich dieselben Prämissen wie in anderen Finalbearbeitungen Gassmanns: Individualisierung im vokalen wie instrumentalen Bereich und ein besonderes Augenmerk auf Spannungsentwicklungen und pointierten musikalischen Höhepunkten, mit anderen Worten das Bestreben, die sich in einem Ensemblesatz bietenden Gestaltungsmöglichkeiten auszuschöpfen.

Dabei arbeitet Gassmann in La cascina regelrecht mit dem musikalischen Material, das Scolari ihm liefert. Auf der einen Seite hat er offenbar keine Skrupel, zu übernehmen, was seiner Ansicht nach gut gelungen ist, und man könnte in der „Pippo caro caro caro“-Melodie durchaus ein Zitat sehen. Zum anderen strebt er aus seinem persönlichen Gusto und seinen Möglichkeiten heraus und trachtet nach Verbesserungen - ein immer wieder von Bearbeitern proklamierter Anspruch, der hier eingelöst wird, indem ein stilistisch bereits etwas überkommenes Ensemble auf den aktuellen Wiener Stand gebracht wurde.

\section{Ein günstiges „Bearbeitungsklima“}

Die Beispiele haben gezeigt, wie sehr der Operndirektor Gassmann im Zentrum der Wiener Bearbeitungspraxis für diesen Zeitraum steht. Grundlegende Eingriffe bei den Finali finden sich vor allem zwischen 1767 und 1770, also in den Jahren nach dem VenedigAufenthalt Gassmanns, zu dem er 1765 aufbrach, als die Wiener Theater aufgrund des überraschenden Todes von Maria Theresias Ehemann Franz Stephan für einige Monate schließen mussten.

Im Sommer 1766 kehrte Gassmann in Begleitung des jungen Antonio Salieri nach Wien zurück und in den folgenden Jahren stand ihm dort ein hochklassiges Ensemble zur Verfügung, an dessen Zusammenstellung er selbst entscheidenden Anteil gehabt haben dürfte. Dieses Ensemble blieb bis 1770 im Wesentlichen stabil mit dem Buffo Francesco Carattoli ${ }^{44}$ sowie dem Sängerehepaar Clementina Baglioni Poggi und Domenico Poggi. Diese Darsteller müssen sich auf der Bühne geradezu blind verstanden haben, denn unter anderem war Clementina Baglioni in Italien als Mitglied der Truppe ihres Vaters Giovanni Baglioni schon vor ihrem Wiener Engagement viele Jahre lang zusammen mit Carattoli aufgetreten.

Zum ,Gassmann-Ensemble der späten 1760er Jahre kamen noch Clementinas jüngere Schwester Rosa Baglioni als Seconda donna hinzu, außerdem die Altistin Teresa Eberardi, der auf Parti serie spezialisierte Antonio Puliti sowie mit den Tenören Filippo Laschi, Giuseppe Pinetti und Gioacchino Caribaldi weitere renommierte Kräfte, die bereits seit

44 Der Römer Francesco Carattoli gehörte schon ab 1764 zum Ensemble und war bei seiner Ankunft in Wien bereits Ende Fünfzig. Er verbrachte die letzten Jahre seiner Karriere in Wien und starb dort 1772, war aber offenbar noch bis recht kurz vor seinem Tod auf der Bühne aktiv. 
Jahren in Italien auf dem Gebiet der Opera buffa reüssiert hatten. ${ }^{45}$ Ein vergleichsweise homogenes Ensemble also, das über mehrere Jahre kontinuierlich mit einem Operndirektor vom Kaliber Gassmanns zusammenarbeiten konnte, mit Sängern, wie Francesco Carattoli, die über großes schauspielerisches Talent verfügten und im Bereich der Opera buffa zu den erfahrensten Künstlern überhaupt zählten, ${ }^{46}$ dazu der junge Salieri, den Gassmann zunehmend als Helfer heranziehen konnte und mit dem ihn ein Lehrer-Schüler-Verhältnis verband, das für beide Seiten befruchtend gewirkt haben dürfte. ${ }^{47}$

Diese Wiener Konstellation der Jahre ab 1766 bot die Möglichkeit zu einer intensiven Art von Teamwork und ein speziell für die Opera buffa günstiges Klima, das zu künstlerischen Herausforderungen reizte. All dies könnte auch eine Erklärung dafür sein, dass bei den Opernbearbeitungen eben auch die Finali so häufig betroffen waren.

Gleichzeitig ergibt sich eine erkennbare Parallele zur persönlichen kompositorischen Entwicklung des Wiener Opernkapellmeisters. Gassmann war als Musiker in Venedig erwachsen geworden, die Libretti Goldonis und ihre Vertonungen etwa durch Galuppi oder Domenico Fischietti hatten ihn von vorneherein geprägt. Durch die erneute Venedig-Reise von 1765/66 konnte er sich vor Ort mit den neuesten Tendenzen innerhalb der Opernlandschaft der Lagunenstadt vertraut machen.

Gassmanns besonderes Interesse an den Ensembles zeigt sich auch in seinen eigenen komischen Opern, die gerade in denselben Jahren entstanden wie ein großer Teil der Wiener Finalbearbeitungen. Insbesondere bei seinen Erfolgswerken L'amore artigiano (1767) und La contessina (1770), die beide auf (älteren) Texten Carlo Goldonis basieren, die von Marco Coltellini für Gassmann überarbeitet wurden, lässt sich dies beobachten. Auch hier kam es zu deutlichen Änderungen bei den Finali, teils mit analogen Verfahrensweisen wie bei den Opernbearbeitungen, so etwa im dritten Akt von La contessina, wo statt des herkömmlichen Liebesduetts und des einfachen Coro finale des ursprünglichen Goldoni-Librettos ein komplexeres Ensemble steht, für dessen Schluss Gassmann ebenfalls auf die Form des Vaudeville-Finales zurückgriff. ${ }^{48}$

Die Finalbearbeitungen erfolgten aus pragmatischen wie ästhetischen Gründen, häufig ist dies nicht zu trennen. Die Diskussion um den Dualismus zwischen theaterpraktischem Denken und künstlerischer Intention wie Ambition kann in Bezug auf das Thema Bearbeitungspraxis nicht als beendet betrachtet werden. Wien bietet für dieses Forschungsgebiet

45 Nebenbei bemerkt war es eben dieses Ensemble, das 1768 eine Rolle dabei spielte, dass Wolfgang Amadeus Mozarts La finta semplice in Wien nicht zur Aufführung kam, denn die Sänger sollen angeblich diese Oper als „nicht theatralisch“ kritisiert haben. Vgl. Stefan Kunze, „,Unvergleichlich komponiert, aber nicht theatralisch'. Komödienstruktur, Rollentypologie und Situation in Mozarts La finta semplice“, in: Mozart e la Drammaturgia Veneta. Bericht über das Colloquium Venedig 1991, hrsg. von Wolfgang Osthoff und Reinhard Wiesend, Tutzing 1996, S. 257-275.

46 Vgl. dazu verschiedene Beiträge von Daniel Brandenburg, insbesondere „Zur Rezeption des BuffaRepertoires im deutschsprachigen Raum. Deutsche Bearbeitungen italienischer Werke in Wien", in: Oper im Aufbruch. Gattungskonzepte des deutschsprachigen Musiktheaters um 1800, hrsg. von Marcus Chr. Lippe, Kassel u. a. 2007 (= Kölner Beiträge zur Musikwissenschaft 9), S. 209-219.

47 Salieri selbst betonte die Wichtigkeit der Aufgaben in der Opernbearbeitung unter Gassmann für seine eigene kompositorische Entwicklung. Vgl. Michele Calella, „Salieris Einlagearien und die Wiener Praxis der Opernbearbeitung", in: Antonio Salieri. Zeitgenosse W. A. Mozarts, hrsg. von Hartmut Krones (im Druck).

48 Vgl. das Vorwort des Herausgebers Robert Haas zur Partiturausgabe von La contessina, Wien 1914 (= DTÖ 42-44) sowie Gustav Donath, „Florian Leopold Gassmann als Opernkomponist“, in: StMw 2 (1914), S. 34-211. 
nicht zuletzt deshalb reichlich Stoff, weil hier hochkarätige Bearbeiter mit besonders hochkarätigen Sängern arbeiteten. Das Bild vom armen Komponisten und Poeten, der sich dem Willen der Sänger stets beugen musste, bedarf der Differenzierung: Standen an einem Theater Kapellmeister wie Gassmann oder Salieri und Dichter wie Coltellini zur Verfügung, so dürfte es die Sänger auch ermutigt haben, sich auf neukomponierte Nummern einzulassen in dem Vertrauen, eine Qualitätsverbesserung gegenüber den ursprünglichen Versionen zu erreichen - als Individuum wie als Kollektiv. Intensive Bearbeitungspraxis, die über das einfache Ersetzen von Arien weit hinausgeht, kann im 18. Jahrhundert auch als Qualitätsmerkmal für einen Opernstandort gesehen werden.

An dieser Stelle lauert die Versuchung, aus dem Dargestellten eine klare Linie herauszuinterpretieren, einen,Wiener Geschmack' zu konstatieren, für den aufwendige Finali schon vor Mozart von großer Bedeutung waren und eine direkte Linie von Gassmann zu Le nozze di Figaro zu ziehen. Es gilt hier jedoch, sich vor Überinterpretationen in Acht zu nehmen. Der häufig vorhandenen Unzufriedenheit mit der traditionellen Opernschlussgestaltung und der nicht allein,Wien-typischen Tendenz hin zum umfassenderen (Final-)Ensemble stehen zahlreiche Gegenbeispiele aus dem Wiener Buffa-Repertoire gegenüber. Viele Bearbeitungen halten an den ursprünglichen Finali fest und somit auch an der traditionellen Nummernfolge gegen Ende. Nicht zuletzt kam es in den Jahren nach 1770 zu einem weitgehenden Verschwinden von Finalbearbeitungen, was damit zusammenhängen dürfte, dass sich zunächst Gassmanns Aufgabenbereich mit seiner Ernennung zum Hofkapellmeister 1772 wandelte und er in den letzten Monaten vor seinem frühen Tod Anfang 1774 kaum mehr Zeit für Opernbearbeitungen gefunden haben dürfte. Sein Nachfolger Salieri zeigte zumindest in den ersten Jahren seiner Tätigkeit als Opernkapellmeister nur wenig Interesse an einer Bearbeitung von Ensembles. Der Blick in die ,Werkstatt Wien' erscheint noch lange nicht abgeschlossen. 